\title{
Entre pujas y facciones: la Cruz Roja Argentina (1864-1914)
}

\section{(4) Adriana Alvarez}

Consejo Nacional de Investigaciones Científicas y Tecnológicas, Universidad Nacional de Mar del Plata, INHUS, Argentina

acalvarmdp@gmail.com

\section{María Silvia Di Liscia}

Facultad de Ciencias Humanas, Instituto de Estudios Históricos y Sociales de La Pampa, Universidad Nacional de La Pampa, Argentina

silviadiliscia@gmail.com

Artículo recibido: 30 de mayo de 2018. Aprobación final: 29 de noviembre de 2018 .

\section{Resumen}

Este artículo analiza el desarrollo de la Cruz Roja Argentina descle su aparición hasta la I Guerra Mundial. Su organización en el país estuvo vinculada a las pujas entre diferentes asociaciones étnicas y grupos de médicos, quienes a su vez se vieron involucrados en las intervenciones y guerras civiles que afectaron a las provincias y a la Capital Federal, entre finales del siglo XIX e inicios del siglo XX. Se trataba una organización internacional cuyo objetivo era la ayuda humanitaria, supuestamente neutral. Los principales actores que llevaron adelante la institución en estos 50 años asumieron diferentes posturas políticas e ideológicas, aunque tenían en común su pertenencia social y la solidaridad masculina. Sus funciones se incrementaron de la atención de víctimas de conflictos bélicos a su cuidado en catástrofes en tiempos de paz, lo cual significó una importante modificación en los principios y en la organización e intervención sociales. A la vez, su interés en las "calamidades" producidas en países vecinos, así como los problemas con los grupos de beligerantes en la Gran Guerra, delimitaron los alcances de esta institución con miras y objetivos internacionales. 


\title{
Between Struggles and Factions: the Argentine Red Cross (1864-1914)
}

\begin{abstract}
This article analyzes the development of the Argentine Red Cross from its appearance until the First World War. Its organization in Argentina was linked to the struggles between different ethnic associations and medical corporations, both involved in interventions and civil wars that affected the inland provinces and Buenos Aires between the late Nineteenth-Century and the early TwentiethCentury. In an international organization whose objective was humanitarian aid and supposedly neutral, the main actors that carried out in this institution during this period assumed different political and ideological positions, although they had in common their social belonging and masculine solidarity. Their functions increased from the attention of victims of armed conflicts to their care in times of peace catastrophes, which meant an important modification in the principles and in the social organization and intervention. At the same time, their interest in the "calamities" produced in neighboring countries, as well as the problems with the belligerent countries during the Great War, delimited the scope of this institution with international goals and objectives.
\end{abstract}

Keywords: Red Cross; State; Argentina; Doctors; Civil War.

\section{Introducción}

A lo largo del siglo XIX, la ciudad de Buenos Aires experimentó un crecimiento diferencial al resto de las ciudades argentinas, profundizado a finales de la centuria fruto de la presencia de inmigrantes europeos que buscaban nuevos horizontes laborales. Ese incremento demográfico inusitado agudizó la precariedad sanitaria, y el hacinamiento habitacional pasó a ser parte de ese nuevo paisaje, marco propicio para la difusión de enfermedades y epidemias, como el cólera o la fiebre amarilla, que diezmaron parte de la población. Las crisis sanitarias engendraron nuevas respuestas gubernamentales, algunas más articuladas que otras, para regular ese "desorden" mediante una serie de dispositivos emanados básicamente de las recomendaciones de la elite médica, que comenzó a intervenir activamente apelando al fortalecimiento de las instituciones sanitarias. En ese sentido partieron varias acciones, como la ratificación del convenio de Ginebra por parte gobierno argentino, a cargo de Nicolás Avellaneda (1879), que habilitó a reconocidos higienistas, como Guillermo Rawson y Toribio Ayerza, a fundar la Cruz Roja Argentina (en adelante, CRA) en 1880.

El presente trabajo tiene como objetivo analizar su formación y actividades principales, dado que se trata de la primera entidad con fines humanitarios de carácter intercontinental. Su naturaleza la diferenciaba del resto de las organizaciones benéficas existentes hasta el momento, por su forma de funcionamiento y organización, puesto que tanto sus competencias como sus modalidades de 
intervención estaban pautadas por protocolos internacionales. Por ello, desde el inicio, sus acciones socio-sanitarias tuvieron un impacto diferente, pues se trataba de una entidad que gozaba (previamente a su aparición formal en el escenario local) de prestigio y reconocimiento general gracias a las tareas realizadas en el continente europeo.

La temporalidad de este estudio se extiende desde los años 1860 -pues se presta atención al proceso formativo de la entidad- hasta mediados de la segunda década del siglo XX, cuando y como consecuencia de la Primera Guerra Mundial, dicha organización inició un profundo proceso de reorganización.

Interpretar los procesos y sucesos que englobaron esta primera etapa de la CRA no es tarea fácil, puesto que por los propósitos que dieron a luz su origen, su pasado permanece en el terreno de las "historias sagradas", como relatos que bajo ningún punto de vista pueden ser sometidos al imperio de la duda (Visacovsky, 2005: 278). Es precisamente ese halo místico que provoca un desafío, dado que pesa sobre esta organización una historia oficial o "tradición inventada" exenta de tensiones, donde abundan triunfos y reconocimientos. ${ }^{2}$

Desde el plano historiográfico, existe una deuda en nuestro medio sobre el estudio y accionar de organizaciones internacionales insertas en el escenario local. En este caso concreto, abundan las historias oficiales pero sin trabajos sistemáticos, y existen nichos documentales hasta la fecha sin explorarse. Además, los estudios europeos y americanos muestran su importancia para la historia de las asociaciones y el humanitarismo médico, entre muchas otras facetas (Hutchinson, 1996; Meyer, 2009). Por ello, resulta de gran interés analizar a nivel nacional una de las primeras instituciones cuyo propósito fue el de actuar como intermediaria neutral en las situaciones de conflicto armado y, a la vez, se constituyó en promotora de las normas del Derecho Internacional Humanitario (Muñoz Blanco, 1999).

Si bien, por un lado, existen trabajos sobre la enfermería y la profesionalización médica en diferentes niveles y contextos y, por otro, hay avances respecto a organizaciones de la sociedad civil, no han sido examinadas en profundidad las conexiones nacionales e internacionales de una asociación de este nivel. ${ }^{3}$ En la historia de la CRA se cruzan de manera compleja varios aspectos, como la consolidación profesional de los médicos, las diferencias y exclusión de género (al tratarse en este período de una asociación formada en su básicamente por varones), el alcance de la intervención médica y social y, por último, de manera más general, las definiciones frente a conflictos internos.

2 Se entiende que una "tradición inventada" puede ser de naturaleza simbólica o ritual, que busca inculcar determinados valores o normas de comportamiento y, en este sentido, la utilización y manipulación del pasado es de vital importancia, ya que "la peculiaridad de las tradiciones inventadas es que su continuidad con éste (el pasado) es en gran parte ficticia”. Hobsbawm y Ranger (2002: 5-7).

3 Al respecto de la primera cuestión, ver Biernat, Ramacciotti et al (2015). En el caso de las asociaciones, llama la atención que en la obra general de Di Stefano, Sábato, Romero y Moreno (2002) exista sólo una mención a la Cruz Roja, en relación a que se trató de una asociación que "mantuvo su forma legal tradicional" a finales del siglo XX, cuando varias se modificaron y pasaron a constituir fundaciones (2002: 259). También resulta curioso que en la obra de Veronelli y Veronelli Correch (2004, Tomo I) no se encuentren referencias a esta institución. 
El artículo está estructurado en cuatro secciones. En la primera se aborda la organización de la CRA en relación a las pujas de diferentes sectores -médicos y no médicos-; en la segunda, se analizan las primeras décadas de esta entidad vinculadas a su primitivo objetivo, que era la intervención humanitaria en conflictos bélicos con la atención de las víctimas. En tercer y cuarto lugar, se explican las nuevas funciones de la entidad en relación a las epidemias masivas y las consecuencias de las catástrofes, y se pone el acento en la composición de la entidad atendiendo a cuestiones ideológicas, profesionales y de género.

Las fuentes provienen de documentación existente en la Biblioteca de la Facultad de Medicina de la Universidad de Buenos Aires, del Centro de Documentación de Madrid de la Cruz Roja Española, de repositorios privados y de la propia institución. En particular, para esta presentación, se analizaron la Primera Memoria presentada a la Asociación Internacional de la Cruz Roja (CR, en adelante) (Roberts, 1880), informes anuales y periódicos de la época, crónicas y libros institucionales (Lozano, 1932) y el Boletín de la Sociedad de la CRA (en adelante, Boletín CRA, serie incompleta: 1891-1908), así como Diarios de Sesiones de la Cámara de Senadores del Congreso Nacional y prensa periódica. Se consideran también publicaciones donde aparecen referencias sobre la Cruz Roja y la información proveniente de las agencias de difusión de la misma entidad en relación a los aspectos históricos, y a su vez diccionarios biográficos para una definición inicial de los perfiles socio-ocupacionales de sus integrantes.

\section{El origen de la CRA}

Al momento de la gestación de la entidad a nivel internacional, ${ }^{4}$ el país se encontraba en el intrincado proceso de convertirse en un Estado y, por tal motivo, no participó de la Convención de Ginebra. Las historias oficiales mencionan que, antes de su reconocimiento formal en 1880, dicha agrupación había desarrollado tareas de socorro en tres episodios relevantes: durante la Guerra con el Paraguay, que involucró a ese país en contra a la Confederación argentina, Brasil y Uruguay entre 1865-1870, y que en el escenario paraguayo significó la muerte y mutilación de soldados, afectando también a la población civil (Manzella, 1980 y 1982).

\footnotetext{
4 La organización inicial de la CR dependió del accionar del empresario y filántropo suizo Henri Dunant, quien inició, a partir de su participación en un conflicto bélico en Italia, un movimiento de sociedades voluntarias de socorro para prestar asistencia a los heridos en tiempos de guerra. Dunant recogió su experiencia en un libro, Recuerdo de Solferino, que se transformó en un clásico para la demostración de la violencia bélica y la necesidad de intervención. Cuatro años después de la batalla de Solferino, y a un año de la publicación, un Comité -integrado por el mismo empresario, el general Dufour, Gustave Moynier y los médicos Théodore Maunoir y Louis Appia- se organizó un Congreso en Ginebra, con la participación de representantes de 16 países y culminó con la firma de un acuerdo en 1864. En ese momento, se recomendó la fundación de sociedades nacionales de socorro, solicitando a la vez la protección y el apoyo de sus gobiernos para mejorar la asistencia a los soldados heridos conflictos bélicos. El Comité formado en dicho Congreso, denominado luego Comité Internacional de la Cruz Roja (CICR), congregó a representantes de diversas naciones para convenir en el establecimiento de sociedades nacionales de socorro como auxiliares de los servicios sanitarios de los ejércitos, y persuadió a los representantes para que aprobasen el primer Convenio. Por ese tratado, los ejércitos tenían la obligación prestar asistencia a los soldados heridos, independientemente del bando al que pertenecían y se introdujo un emblema uniforme para los servicios sanitarios: una cruz roja sobre fondo blanco. Aguirre Reyes y Arrizabalaga (2016).
} 
También actuaron en la epidemia de fiebre amarilla de 1871 en Buenos Aires, que diezmó a la población, y en la epidemia de cólera a mediados de los años 1880. ${ }^{5}$ Durante estos tres sucesos, que incluían asuntos bélicos, movilización de tropas y organización sanitaria al mismo tiempo, hubo intervención de la CR, pero no de manera formal.

Los hechos anteriores, que involucraban a distintos países latinoamericanos en un conflicto bélico de singular importancia o a epidemias difíciles de combatir con la tecnología médica del siglo XIX, no impusieron la organización sistemática en el país, sino que tal cuestión se planteó a partir de problemas internos e involucró a otras asociaciones étnicas. El presidente Nicolás Avellaneda, quien gobernó a la Argentina entre 1874-1880, inauguró su gestión en medio de una revolución, en un marco caracterizado por los periódicos de la época como de "grave situación social”. Desde algunos medios, como el Diario España Moderna (propiedad de José Paul y Angulo ${ }^{6}$ ) se planteó justamente la necesidad de crear una filial de la CR a fin de auxiliar a las víctimas de los sucesos revolucionarios. A partir de este episodio, varios ciudadanos constituyeron el Comité Sudamericano, cuyo presidente fue Antonio P. Aleu ${ }^{7}$, propietario del Diario El Español (García Sebastiani, 2010:56). Ese comité, una vez finalizados los sucesos revolucionarios, confeccionó un proyecto de reglamento, sometido a la aprobación del gobierno nacional de entonces, sin obtener confirmación oficial. ${ }^{8}$

Recién frente a las jornadas revolucionarias del 20 y 21 de junio de 1880 se evidenció la necesidad de relanzar la entidad. Su creación fue caja de resonancia de las pugnas existentes entre una corporación médica en rápido ascenso y la consolidación en el entramado político institucional de entonces, auxiliado por sectores civiles nucleados en las entidades de beneficencia que no estaban dispuestos a ceder terreno. El examen del proceso demuestra cierta oposición entre médicos y entidades étnicas de beneficencia, secundadas por empresarios e intelectuales influyentes, como los ya mencionados Aleu y Angulo.

Al repasar puntualmente los sucesos es posible describir la aparición y desarrollo de esos campos en pugna, que se unen a procesos políticos más amplios. Al término de la presidencia de Avellaneda (1874-1880), el problema de la sucesión presidencial conllevó a la separación de dos grupos: por un lado, la conjunción oficialista de Partido Autonomista Nacional (PAN), que impulsaba a Julio Argentino Roca y proponía federalizar la ciudad de Buenos Aires, para que la provincia de Buenos Aires dejara de ser la anfitriona de la sede del gobierno nacional. Por

5 (11 de septiembre de 1963). La Nación, Buenos Aires.

6 Procedente de una familia adinerada y educado en Londres, José Paul y Angulo fue ferviente simpatizante de la causa republicana en España. Por problemas políticos y policiales emigró a Buenos Aires, donde fundó un periódico. Flores García (1916: 3).

7 Nacido en Barcelona en 1847, Aleu emigró a Buenos Aires, donde se recibió de abogado en 1869 y residió hasta su muerte, en 1926. Su intensa actividad como promotor de iniciativas catalanistas incluyó la fundación y dirección de L'Aureneta, primer periódico de América del Sur escrito en catalán, publicado de manera intermitente entre 1876 y 1892. Fue presidente y dirigente de algunas de las principales asociaciones panhispánicas, como la Patriótica Española, el Club Español y la Sociedad Española de Beneficencia. Fernández (2016: 89-90).

8 Acta de la Sesión, 9 de Junio de 1880. En Roberts (1880: 49). 
otro lado, se fortalecían los autonomistas de Bartolomé Mitre, quienes no aceptaban a Roca, puesto que entendían que retornaría el dominio de los "caudillos feudales" del interior sobre la "progresista y liberal" ciudad porteña de Buenos Aires. Así, hacia 1880, ciertos grupos y personalidades intentaron consolidar el gobierno central, exaltando el papel del orden y la paz frente al caos introducido por las facciones localistas (Gallo, 1992). Mientras Roca contaba con el respaldo de la mayoría de los oficiales del ejército nacional, y la Liga de Gobernadores le garantizaba el apoyo de casi todas las provincias, tanto Mitre como el gobernador de Buenos Aires, Carlos Tejedor, y sus seguidores, comenzaron a armarse con el fin de preparar una insurrección, que para el mes de junio de 1880 ya era un secreto a voces. La revuelta armada estalló en Buenos Aires entre el 16 y el 21, dejando, tal cual se preveía, centenares de heridos. ${ }^{9}$

En los días previos a esos enfrentamientos, y mientras en ambos bandos se aprestaban con febril actividad los recursos bélicos, los médicos organizados en el Circulo Médico Argentino (CMA, en adelante), la Asociación Médica Bonaerense (AMB, en adelante) y la Sociedad de Farmacia (SF, en adelante), convocaron a una reunión con la idea de formar la CRA, que finalmente se llevaría a cabo el 8 de junio de 1880. Allí se eligió una junta provisoria y se sancionó un primer reglamento. Dicha junta estaba constituida por médicos en su totalidad; la presidía Guillermo Rawson, secundado por Bartolomé Novaro y Alberto Finch y participaban también Juan Golfrini, Antonio Crespo,José M. Jorge, Tomás Ballestra, Pedro Defoix, Pedro Roberts, Carlos Lloveras y Lucio Meléndez..$^{10}$ Se trataba de un grupo de médicos que desde hacía años compartían espacios asociativos diversos. Tal era el caso de Golfrini, quien junto otros facultativos, como José Eduardo Wilde y Pedro Mallo, y la protección de Guillermo Rawson, habían participado de la AMB (González Leandri, 1999:195). También el de Antonio Crespo, Bartolomé Novaro (vicepresidente y secretario) y José Jorge, quienes habían compartido en 1875 la fundación del CMA junto a José María Ramos Mejía. Esta institución es considerada como el antecedente de la Asociación Médica Argentina (Fustioni, 1971:130).

Tanto el CMA como la AMB eran referentes del quehacer médico nacional y cobraron notoriedad pública en el momento de su aparición, generando debates hacia adentro de una organización médica hasta entonces jerárquica y casi monolítica. José Jorge y Antonio Crespo eran los primeros líderes que, junto a Ramos Mejía, tuvieron una actuación destacada en los eventos de 1873, donde se impugnaron a los catedráticos dirigentes y se plantearon la necesidad de reformas radicales en los reglamentos y planes de estudio de la Facultad de Medicina de la Universidad de Buenos Aires, la única por entonces formadora de profesionales en todo el país. Un par de años después organizaron el CMA, concebido como una institución de nuevo cuño y de "características cuasi revolucionarias" (González Leandri 1999: 194-195). Esta última era una organización que aglutinaba a estudiantes y graduados recientes, con opiniones enfrentadas al grupo de médicos a cuyo cargo estaba la dirección de la Facultad de Medicina. El principal órgano de difusión de sus

9 Sobre el conflicto ver Sábato (2008).

10 Acta de la Asamblea, 8 de Junio de 1880. En Roberts (1880) 
ideas fueron los Anales del CMA, cuyos columnistas más referenciados eran José María Ramos Mejía, Antonio Crespo, Bartolomé Novaro y Gregorio Aráoz Alfaro, entre otros (Souza, 2006: 107-108). Es interesante destacar que todos ellos figuran en los anales de la institución como miembros fundantes de la CRA (Manzella, 1980: 25), jóvenes profesionales e higienistas que impugnaban las instituciones médicas y procuraban diferenciarse de la "masa de médicos comunes, a la que sus discursos implacables arrojaban al borde de la profesión” (González Leandri, 1999: 230).

Dos higienistas con trayectoria, como Emilio Coni y Guillermo Rawson, habían promovido la $\mathrm{CR}$ en virtud de la representación internacional y del afán de la Argentina de ingresar ideológica y científicamente al concierto de las naciones occidentales. En 1884 Emilio Coni fue Delegado de la República Argentina y de la CRA en la Tercera Conferencia Internacional de las Sociedades de la CR en Ginebra (Coni, 1918). ${ }^{11}$ Rawson tuvo gran incidencia en la modernización sanitaria y el control de las epidemias, así como en la naciente estadística del Estado argentino y como ministro del interior impulsó el primer censo nacional, levantado en 1869. Fueron notorias sus conexiones académicas a nivel internacional, tanto en Estados Unidos como en Francia (Rawson, 1891, Tomos I y II). ${ }^{12}$

Por lo tanto es muy posible que el interés de esos profesionales en estar al frente de la creación de la CRA haya sido paralelo al proceso de búsqueda y reafirmación generacional y profesional, en cual se encontraban desde la década de 1870, y que era cuestionado por los "otros médicos". De hecho, esa primera formación de la CRA a la que aludimos con anterioridad, relacionada con las jornadas de junio de 1880, fue sutilmente cuestionada y no prosperó. Inmediatamente luego de constituida, y de la difusión periodística sobre el acto realizado en la sede de la $\mathrm{AMB}$, se recibieron una serie de notas, una de ellas proveniente de Angulo. En esa misiva se informaba que la idea de fundar la CRA había sido de la Sociedad Española de Beneficencia, la cual contaba con el apoyo del asociado español Francisco Ibarra, delegado en suelo argentino de la sección española de la CR. Por esta razón, y a pesar de lo realizado en la AMB, se convocó a una sesión popular para el día siguiente en los Salones del Club Español, a efectos de constituir la sección nacional la $\mathrm{CR}$, a la cual se invitó a la $\mathrm{AMB}$, el CMA y $\mathrm{AF}^{13}$

También envió una nota Antonio P. Aleu, donde refería que había tomado conocimiento por los periódicos de lo acaecido el 8 de Junio en la sede de la AMB, e informaba que desde 1874 existía en la ciudad de Buenos Aires el Comité Internacional

11 Este prestigioso higienista, nacido en Corrientes en 1854, fue fundador y director de la inspección médica e higiénica de las escuelas públicas de Catedral Sur y San Telmo; fundó la oficina estadística municipal de Buenos Aires y dirigió Los Anales de Higiene Pública. En 1892 fue director de la Administración Sanitaria y Asistencia Pública de Buenos Aires; también dirigió la primera maternidad municipal del hospital San Roque, el primer asilo nocturno municipal y fue el creador del Patronato de la Infancia y Asistencia a la Infancia. Su amplia labor también implicó a asociaciones organizadas para luchar contra las enfermedades sociales, ya que dirigió los dispensarios de la Liga Argentina contra la Tuberculosis. Alvarez (2007: 8).

12 Existen varias biografías sobre esta figura, entre las cuales se destaca Larrain (1895); Carbonell (1953) y Martínez (1891).

13 Nota de Juan Paul Angulo. Roberts (1880: 45). 
Sub-americano de la CR, cuya Comisión Directiva él presidía. Además adhería a la convocatoria que se estaba realizando en el Club Español (del cual había sido presidente) y aclaraba que le resultaba innecesario y hasta cierto punto perjudicial la constitución de otro centro con tendencias y propósitos iguales. ${ }^{14}$

Las dos notas enviadas por sendos españoles, de cierta influencia y prestigio tanto periodístico como en el escenario de las asociaciones étnicas, hicieron desistir de la propuesta original al grupo recientemente constituido sobre la base de un perfil profesional. Se dejó sin efecto esa primera Comisión, y los facultativos se sumaron a la reunión "popular" realizada por la Sociedad Española. El grupo de médicos se vio acorralado, razón por la cual acordaron formar una nueva Junta Ejecutiva que representara a diferentes sectores, con la inclusión no sólo de médicos sino de abogados, comerciantes y empresarios, que tendió a respetar un equilibrio de sectores, más que el espíritu corporativo que había priorizado la primera de las juntas. De ahí entonces que este Consejo Supremo de la CRA tuviera dos presidentes honorarios: Guillermo Rawson, representante de la corporación médica, y el médico español Toribio Ayerza, por la Sociedad Española. ${ }^{15}$

Este hecho marca diferentes cuestiones que explican el desenlace de la primera fundación de la CRA: ante todo, eran tiempos de reconfiguraciones del saber médico con un reordenamiento de los estilos ya existentes, donde a nivel local varios de los actores que asumieron la primera fundación de la CRA (Rawson, Crespo, Jorge, Novaro, Golfrini y otros) buscaban posicionarse como referentes de la medicina clínica y de una renovada agenda de trabajo. ${ }^{16}$ Posteriormente, y vinculado con lo anterior, tal situación se enmarcaba en las tensiones y conflictos que atravesaban a la corporación médica, iniciados en la Facultad de Medicina, y que alcanzaban a la opinión pública. Su resultado fue el debilitamiento de la elite médica tradicional y el avance de nuevos actores, como José María Ramos Mejía, que había apoyado la iniciativa de los médicos que encabezaron esa primera fundación frustrada de la entidad humanitaria.

En ese contexto, la CRA constituyó un espacio de disputa entre esos profesionales y los que estaban forjados en una tradición anterior, cuyos modelos quedaban marcados por sus vínculos con entidades de beneficencia. En esa presión por lograr monopolizar el espacio técnico-médico y profesional, la iniciativa de fundar la CRA puede ser analizada como parte de estrategias alternativas desplegadas por los sectores subalternos que llegaron a la impugnación de las elites dominantes y a intentos de usurpación de algunas de sus atribuciones (González Leandri, 1999: 32). Dicha circunstancia no trajo aparejado el dominio de la corporación médica o incluso el control sobre las organizaciones impulsadas como fue la CRA, lo que delata que se trató de un proceso de pugnas y, a la vez, de negociaciones entre ambas partes.

14 Nota de Antonio P. Aleu. Roberts (1880: 45).

15 Toribio Ayerza nació en España, y cuando arribó a Argentina ya era médico. Fue socio fundador de la Asociación Española de Socorros Mutuos y presidente de 1861 a 1862 y de 1870 a 1871 . Pesqueira (1919: 9-36).

16 Sobre el alcance científico, ver Fleck (1989). 
Lo expresado anteriormente explica las razones por las cuales la primera fundación de la CRA fue desarticulada no sólo por otros profesionales, sino por miembros poderosos de la elite socioeconómica que desde épocas remotas tenían incidencia en la dirección de entidades benéficas sanitarias. Ahora bien, tampoco este sector podía simplemente prescindir de los médicos que gozaban para ese entonces de respeto profesional, como era el caso de Guillermo Rawson, o de una pluma filosa como la de José María Ramos Mejía, así como de la influencia de José Jorge o Antonio Aleu, entre otros, por lo que se vieron obligados a negociar la refundación de la CRA y una nueva composición de la Comisión Directiva, donde tuvieron lugares destacados.

Una vez organizada la CRA, comenzó de inmediato a intervenir en la atención de heridos producto de los conflictos armados del período, tarea que, como se verá a continuación, al igual que su fundación estuvo cruzada por las antinomias del momento.

\section{La neutralidad puesta en jaque}

El largo camino hacia el reconocimiento legal de la CRA plantea conos de sombra respecto a su imparcialidad política. Ciertas sospechas de la que fue objeto durante ese primer momento contribuyeron a demorar la instalación de la legitimidad de su presencia en estas tierras y su tardío reconocimiento legal. Como indicamos, Avellaneda aprobó el Primer Convenio de Ginebra en 1879, y luego de superados algunos conflictos internos, la entidad se fundó el 10 de junio de 1880. No obstante, recién en 1890, bajo la presidencia de Carlos Pellegrini, se le otorgó la personería jurídica (Manzella, 1982: 292).

Este retraso de diez años tiene un correlato político que vamos a observar más cuidadosamente. Luego de la aprobación formal a través del Poder Ejecutivo en junio de 1880, el nuevo Consejo de la CRA se dirigió al Gobierno de la Nación y de la Provincia de Buenos Aires solicitando el reconocimiento de su existencia. Por entonces, se vivía una tensa situación entre ambos, bajo la representación de Nicolás Avellaneda y Carlos Tejedor, respectivamente. A través de Benjamín Zorrilla, Ministro de Guerra y Marina de la Nación, Avellaneda reconoció a la CRA y prestó acuerdo para su instalación en la ciudad de Buenos Aires. Con esta resolución, se autorizaba el uso a esta asociación del símbolo de identidad. ${ }^{17}$ Pero faltaba la declaración del gobierno de la Provincia de Buenos Aires, reconociendo las prerrogativas de los miembros de la CRA en casos de conflicto entre ambas partes. Las dilaciones se debieron a que el Cuerpo de Sanidad Militar de Buenos Aires (CSMBA), interpretaba "en el sentido más favorable a sus deseos el texto de las bases del Tratado de Ginebra, creyéndose autorizados para ampararse con los distintivos de la neutralidad o sea de la Cruz Roja” (Roberts, 1880: 11). El Consejo Supremo de la CRA veía en esto un peligro, porque dos entidades utilizaban el 
mismo signo, por lo que le solicitaron a dicho cuerpo su abandono o el uso de algún otro distintivo que diferenciase a los miembros del CSMBA de los que pertenecían a la CRA. Luego de varias negociaciones, ese sector militar acordó usar entonces el brazalete en la botamanga y un galón de oro en la gorra (Roberts, 1880: 12).

A su vez, la resistencia a la autorización por el gobierno provincial también incluía otros factores. Así, el gobernador Tejedor y su Jefe de Milicia, Martín de Gainza, establecieron que si bien entendían que "Las sociedades de Caridad dedicadas al alivio y cuidado de los heridos, son altamente recomendables (...) la caridad no puede llevarse hasta proteger al enemigo con los recursos del pueblo de Buenos Aires" ${ }^{18}$ También indicaron que con el fin de impedir abusos "que pueden cometerse a la sombras de las instituciones de caridad (...) ningún hombre obligado al servicio militar podrá formar parte de las Asociaciones de Caridad, sin previa autorización del Ministro de Milicias". ${ }^{19}$ Por lo tanto, los decretos firmados por el Gobernador Carlos Tejedor desconocían el carácter neutral de la CRA, y a consecuencia de tales decisiones, durante los sucesos revolucionarios que tuvieron como correlato la federalización de Buenos Aires, esta entidad no pudo brindar servicios al Hospital de Flores ni a las zonas de Luján y Belgrano (Roberts, 1880: 27).

Las razones de la postura del gobierno provincial pueden ser explicadas por quienes componían la CRA, encolumnados en uno de los bandos. Tal era el caso de su presidente, Guillermo Rawson, ex mitrista, visto con cierto recelo por parte del Gobernador Tejedor, dado que las fuerzas de ese sector eran ahora su principal sustento. Por lo tanto, se dudaba de la neutralidad de una entidad cuyos principios estaban sustentados por la prescindencia política. A la vez, puede sospecharse que el inmediato reconocimiento de las autoridades nacionales al pedido de la CRA implicaría el movimiento inverso, pues se trataba de facciones aliadas al gobierno central. Tal cuestión cobraría solidez unos años después, al aprobarse la Ley Nacional № 2.676, que más adelante retomaremos.

A pesar de las limitaciones impuestas por el gobierno de la Provincia de Buenos Aires, los miembros de la naciente CRA se abocaron a prepararse para los sucesos bélicos, solicitando elementos de auxilio y curación a los hospitales organizados por las asociaciones étnicas, únicos que funcionaban en el ámbito porteño con cierta eficacia. El Hospital Español, el Italiano y el Francés donaron enseres, camas y espacios en sus edificios para la tarea que realizaría en esos días la naciente CRA, de manera tal que en la jornada del 20 de Junio se dedicaron al transporte "de los heridos en catres, carruajes en zorras y aun a hombro por la falta de elementos de traslación, jóvenes, ancianos, ricos y pobres, artesanos y hombres de letras buscaban ávidos ocasión de prestar servicios a los heridos” (Roberts, 1880:17). En los combates de Corrales, Puente Alsina, Puente de Barracas, donde las fuerzas del general Levalle ${ }^{20}$ contaban con escasos auxilios, obtuvieron los refuerzos de

18 Decreto Provincial, 23 de Junio 1880. Roberts (1880: 59).

19 Decreto Provincial, 23 de Junio 1880. Roberts (1880: 59).

20 El General Nicolás Levalle participó en la Guerra de la Triple Alianza y fue uno de los jefes de la denominada "Conquista al desierto", fundador del Club Militar (actual Círculo Militar), Jefe del Estado Mayor General del Ejército, y tres veces Ministro de Guerra y Marina de la Nación, bajo las presidencias de Miguel Juárez 
la flamante entidad. Los médicos Pedro A. Pardo, Toribio Ayerza, Lucio Meléndez, Pablo Marengo, José María Ramos Mejía, Julián Fernández, Ignacio Pirovano y Juan Argerich brindaron su asistencia (Manzella, 1980: 15). Se instalaron hospitales de sangre en el Colegio de El Salvador, en el Hospital Español y en el Hospicio de Las Mercedes, éste último un centro de atención para enfermos psiquiátricos (Lozano, 1932: 19). A pesar de estos esfuerzos, no pudieron avanzar más allá de la ciudad de Buenos Aires, dado que en el espacio provincial tenían impedido ejercer, y esa desconfianza sin duda iba a contrapelo de las nociones generales de ayuda humanitaria.

Desde el punto de vista de los aportes de la CRA a la sanidad militar, se destacaron los hospitales de sangre, instalaciones sanitarias provisorias ubicadas en cercanías de la zona de combates y destinadas a la asistencia de heridos. Estos hospitales no eran en sí algo novedoso ya que se utilizaban en Europa, e incluso en el país se habían instalado en décadas anteriores en las campañas militares del General San Martín (Eleta, 2017: 32). Pero lo distintivo y singular es que, en el caso analizado, fueron ubicados en nosocomios del centro porteño. Estos hospitales de sangre aceleraban -aun en el momento de combate- la llegada de los heridos a los nosocomios y evitaban una mortalidad mayor. Para esta tarea, la CRA contó con " 41 catres nuevos, 14 catres viejos, 2 catres de campaña, 4 camillas para los heridos, y 21 palos para camillas que fueron improvisadas con telas gruesas” Roberts (1880: 91).

Según los informes, las mejoras tuvieron importante papel en los sucesos bélicos, pues las comisiones y las ambulancias de la CRA asistieron a 319 heridos, tanto en hospitales como a diferentes domicilios particulares. ${ }^{21}$ En este caso, se colaboró en el cuidado de los heridos con las Damas de Beneficencia, las Beneméritas Damas del Socorro y los cuerpos médicos de los hospitales. Pero, según la documentación, el hecho de haber sido los heridos "recogidos por el personal de la Cruz Roja muy luego de ser heridos, ha contribuido en la mayor parte de los casos a un resultado favorable en la curación" (Roberts, 1880: 68). ${ }^{22}$

Ahora bien, según la crónica de la entidad, el mayor reconocimiento social no se produjo particularmente en ese momento, sino a partir de su participación en los sucesos enmarcados en la crisis de 1890 (Cruz Roja Argentina, 1919:5).Hacia fines de los años 1880, la expansión económica argentina comenzó a mostrar cierta debilidad, con un aumento de la deuda contraída por la entrada y la detención del flujo de capitales externos producto de la crisis europea. A nivel socio político,

Celman, Carlos Pellegrini y José Evaristo Uriburu. Comandó las tropas leales que vencieron en la Revolución del Parque de 1890 y mantuvo estrecha relación con Julio Argentino Roca. Piccinali (1981).

21 En el detalle se indicaba el traslado de 36 víctimas al Hospital de sangre (Calle Paraguay), 31 al Hospital de Mujeres, 6 al hospital de Hombres, 47 al Hospital de Aduana Lanús, 30 al Hospital de Convalecencia, 28 al Hospital Español, 31 al Hospital Italiano, 23 al Hospital francés, 17 al Instituto Frenopático, 23 al Hospital de la Caridad y 21 al Hospital del Salvador. También se llevaron pacientes a domicilios particulares: 8 a Calle Salta Nro. 174, 2 a la Calle Concepción Nro. 18 (Casa del Señor Loezsich) y un herido a la Calle Zeballos Nro. 115. En Roberts (1880: 67).

22 En relación a los aspectos técnicos, se reconoce que la CR impulsó determinados cambios para mejorar el traslado de heridos y la sanidad en el campo de batalla: ver al respecto la postura de un miembro de la CR española, el médico Nicasio Landa (2016). 
aparecieron diferentes propuestas, tanto en las filas del oficialismo como en los distintos grupos de oposición, que bregaban por un espacio político. Producto entonces de reclamos y contradicciones, estalló el 26 de julio de 1890 lo que se conoce como la "Revolución del Parque".

La oposición al gobierno conservador del entonces presidente Miguel A. Juárez Celman se atrincheró en el Cuartel del Parque de Artillería y desde allí iniciaron los combates, que duraron tres días. En las filas de los revolucionarios había figuras como Leandro N. Alem, Lisandro de la Torre, Aristóbulo del Valle, Hipólito Yrigoyen, Nicolás Repetto, Emilio Mitre (hermano de Bartolomé), Marcelo T. de Alvear y Juan B. Justo. En el plano institucional, la Revolución provocó la renuncia del presidente y su reemplazo por Carlos Pellegrini.

En dichas circunstancias, los heridos del Parque de Artillería fueron trasladados en camillas al Hospital de Clínicas, con la asistencia de la CRA. En el informe elevado por la institución a Ginebra, se señalaba el establecimiento de puestos de auxilio y la recepción de donaciones, confinadas en depósitos. Además, se proveyó una ambulancia de auxilio en el Convento de San Francisco, dotado de cuatro salas con 94 camas para heridos que fueron asistidos por personal médico, practicantes y civiles (Caras y Caretas, 1901). Se informó que:

bastó para que los carros de la Empresa Villalonga, llevando la bandera de la CRA, recorriesen algunas cuadras, solicitando la cooperación de los vecinos, para que éstos los Ilenasen de ropas, colchones y demás artículos requeridos para los hospitales y ambulancias. Tratándose de los heridos, la liberalidad de la población no ha conocido límites, y, merced a tan generoso como espontáneo impulso, pudimos establecer varios hospitales de sangre en pocas horas. Argentinos y extranjeros apoyaban resueltamente las disposiciones y medidas adoptadas por nuestra institución, y acudían a prestar su concurso, ofreciéndonos sus servicios como médicos y asistentes, u ocupándose en llenar cualquier necesidad (Lozano, 1932: 21).

Según Caras y Caretas, a partir de los sucesos revolucionarios se abrió un nuevo período en la historia de esta institución, puesto que los poderes públicos realizaron un reconocimiento de su labor y, como contraparte, recibieron el apoyo gubernamental para terminar de definir su organización interna. ${ }^{23} \mathrm{Al}$ término de la revolución, el Consejo Supremo dirigió una nota al Ministro de Guerra, General Levalle, solicitándole se tomaran medidas con la finalidad de proteger títulos y emblemas de la CRA, y "reprimir el uso abusivo de sus insignias y su nombre, tanto en tiempos de paz como de guerra". El pedido fue apoyado mediante la sanción de la Ley Nacional № 2.676 en 1890, con la presentación de dos diputados que eran también médicos: Osvaldo Magnasco y Bartolomé Novaro. Este último había sido en junio de 1880 miembro fundador de la Asociación de la CRA, con el cargo de vicepresidente primero y luego presidente del CMB, entre 1881 y $1883 .{ }^{24}$

23 (10 de agosto de 1901). Caras y Caretas, Buenos Aires, Año IV, núm. 149.

24 Según el Acta de Fundación en la Sesión Constitutiva del 10 de junio de 1880, Bartolomé Novaro presidió la sesión e impulsó la constitución del primer Consejo Supremo de la sección Argentina de la Cruz Roja. Manzella (1980: 16). 
En el debate parlamentario sobre dicha legislación pueden encontrase explicaciones a las reticencias que unos años antes había tenido el gobierno de la provincia de Buenos Aires en reconocer a la CRA como una entidad humanitaria y neutral. Se denunció en el recinto parlamentario el uso inescrupuloso de la bandera de la CRA puesta al servicio, por ejemplo, del transporte de municiones o como forma de penetrar en el terreno rival de manera ilegal, no para realizar acciones de auxilio de heridos, sino de espionaje (Lozano, 1932: 25).

Dos años después, la entidad parecía haber logrado cierto reconocimiento de las entidades oficiales. Sus opiniones fueron vertidas de forma institucional mediante un informe fechado en Julio de 1892, meses antes de producirse la renuncia de Guillermo Udaondo en el Departamento Nacional de Higiene (máxima autoridad sanitaria en el país) y su reemplazo por José María Ramos Mejía (uno de los impulsores fundacionales de la CRA), quien permaneció en el cargo hasta 1898, cuando fue reemplazado por Eduardo Wilde. ${ }^{25}$

Por entonces, la CRA propuso a las autoridades gubernamentales que en caso de disturbios armados o guerra, se colocaran bajo el amparo de su bandera los edificios y maquinarias de las obras de salubridad, con el fin de proteger a los afluentes cloacales y el agua potable de posibles destrucciones como efecto de los combates. Se argumentaba que su rotura podía contaminar las aguas y así difundir enfermedades como, por ejemplo, el temido crup. ${ }^{26}$ También se solicitaba que se exceptuara del servicio militar al personal tanto de las obras de salubridad como de la CRA, pues los dirigentes entendían que su rol era tan o más importante en dichas instituciones, que en las filas de los ejércitos (Cantón, 1892: 7-9). Este punto, en un país donde se estaba debatiendo la defensa de la patria como obligación entre los varones (Ley aprobada en 1901), es sin duda un aspecto importante respecto de una asociación que ponía sobre el tapete principios contradictorios respecto a la guerra.

A finales del siglo, la CRA también asistió a los heridos en la revolución de 1893 en Santa Fe, y de la revolución de Entre Ríos en 1899 (Lozano, 1932: 34). En este último caso, se organizaron equipos de sanidad formados por médicos, practicantes, farmacéuticos y enfermeros, al tiempo que un grupo de damas, bajo la dirección de la primera médica argentina, Cecilia Grierson, se ocupaba de confeccionar útiles de curación en el mismo local de la CR. Para entonces, la CRA parecía tener clara aprobación pública y social, aunque las mujeres mantenían un lugar poco destacado. ${ }^{27}$

Hacia 1907, un movimiento armado en la provincia de San Juan llevó a que un grupo de damas organizara la atención de los heridos en nombre de la CRA, con el inmediato apoyo de la filial de la Capital Federal. El presidente, Tomás

25 Tanto Ramos Mejía como Eduardo Wilde compartieron su cercanía a la CRA y además fueron activos miembros de la masonería argentina. Lappas (1958).

26 Nombre popular que en la nosología de la época definía a la laringotraqueobronquitis, seria infección viral que afectaba sobre todo a los niños.

27 (10 de agosto de 1901). Caras y Caretas, Buenos Aires, Año IV, núm. 149. 
de Veyga -médico-, y todos los integrantes, enviaron parabienes a las mujeres, que "han demostrado poseer en el más alto grado las virtudes de abnegación y caridad que son el esplendor y la gloria de la mujer argentina" ${ }^{28}$. En una breve carta, el mismo presidente indicaba que las víctimas en la lucha de las triunfantes fuerzas revolucionarias contra el gobernador habían sido atendidas en los domicilios, puesto que se carecía de instituciones respectivas en San Juan, pero se había autorizado a las damas a utilizar el emblema de la CR. ${ }^{29}$ Esta situación dio pie para que se organizara en la provincia cuyana un comité, inusualmente presidido por mujeres. ${ }^{30}$

Al igual a lo ocurrido en otras Sociedades, la CRA no sólo centró su accionar en la asistencia de soldados heridos según lo contemplado por la Convención de Ginebra. Ese marco jurídico se vio desbordado por nuevas demandas de actuación humanitaria (Arrizabalaga, 2016: 825). La proliferación de epidemias, inundaciones, terremotos, sequías y hambrunas, que afectaban al conjunto de la sociedad civil, será la actuación -en el plano no bélico- que abordaremos a continuación.

\section{Las nuevas funciones de la CRA: de epidemias a catástrofes}

Paralelamente a la atención de las víctimas por conflictos internos, entre 1886 y 1887 la CRA se ocupó de las víctimas del cólera en Tucumán, Mendoza, Jujuy y Salta. ${ }^{31}$ A la asistencia agregaron acciones preventivas, además de la publicación de folletos profilácticos, el dictado conferencias y la instrucción inicial de enfermeros (Lozano, 1932: 20). Las acciones de socorro en el interior argentino estimularon la formación de Comités -como el de San Juan analizado en el apartado anterior- aunque de manera embrionaria, pues tardaron en ejercer como organismos activos y con autoridades constituidas.

En 1886, la naciente filial de la CRA en Tucumán anunciaba: "una calamidad pública pesa sobre tres ciudades argentinas, y aunque no ha tomado aún, por fortuna, proporciones tales que pueda considerarse inevitable o inminente su propagación a las demás poblaciones de la República, el peligro de la invasión existe" (Araoz Alfaro: 1938). Al mismo tiempo, se apelaba a las autoridades a

28 Boletín de la CRA, 1907, Serie III, núm 4: 3.

29 Sobre el contexto general de estos movimientos en las provincias, ver Lobato (2000). La revolución contra el gobernador conservador Manuel José Godoy tuvo el apoyo de partidarios porteños, quienes enviaron armas y pertrechos al candidato triunfante, Carlos Sarmiento. En el intercambio epistolar entre las damas sanjuaninas y el Comité central, se percibe también cierto apoyo a uno de los bandos, en la medida que se indica que "Desde los primeros momentos, los doctores Alejandro Quiroga y Luis Palacios y el farmacéutico Sr. Roberto Sarmiento ofrecieron sus servicios a nombre de la Cruz Roja, en el Hospital Rawson, donde se atiende la mayoría de los heridos. El día 8 la Señora Presidenta, Teléfora de Sánchez, y la vice que suscribe (en ejercicio de la presidencia), entregaron al Dr. Aguilar presidente de la Comisión de Hospitales, la cantidad de $150 \$ \mathrm{~m} / \mathrm{n}$ y útiles y curaciones a nombre de este Comité". La Sra. Sánchez recibió un herido en su casa, al igual que la socia Amalia Cambas; las socias visitaron a los heridos. El comité sanjuanino no disponía por entonces de un local, pero poco después de la revolución presentó los planos de la sede. Boletín de la CRA, 1907, núm. 4: 21-22.

30 Boletín de la CRA, 1907, Serie III, núm. 4: 4.

31 Sobre la epidemia y su impacto en el interior argentino, ver Fernández (2017: 19-20) y Prieto (1996: 61). 
mejorar las condiciones higiénicas de la ciudad y al pueblo a hacer " suyas las obligaciones que nos impone el interés de velar por la conservación de la salud pública" y se solicitaba la donación de "ropas y camas para los pobres", advirtiendo que "mañana quizás tendremos que pedir pan para los hambrientos". ${ }^{32}$

Otra iniciativa formalizada en el interior del país fue el Comité de Córdoba, constituido a partir del auxilio recibido para paliar las inundaciones de 1891. Bajo la presidencia de Augusto López, se agrupó a "caracterizadas personalidades de la ciudad universitaria”. La organización cordobesa fue comandada por facultativos varones y secundada por un núcleo de damas pertenecientes a las más encumbradas familias de aquella provincia, como veremos en el siguiente apartado. ${ }^{33}$

Hacia 1890, la CRA había asistido a heridos en combates e intervenido en epidemias de alcance masivo y alta morbi-mortalidad, como el cólera. Hasta ese momento, y dada la cantidad de médicos aglutinados, esta entidad era eslabón clave en la atención médica. A la vez, no colisionaba con las múltiples acciones llevadas a cabo por el resto de las entidades de beneficencia en la atención a madres, huérfanos, discapacitados y enfermos psiquiátricos, por nombrar los principales conjuntos sociales afectados.

Sus acciones se ampliaron hacia las "calamidades", por lo cual se desplegó un discurso conciliador para con el resto de las entidades existentes, ubicándose como auxiliar de las tareas realizadas por las organizaciones benéficas. Uno de los primeros hechos en este sentido fueron, en 1891, las inundaciones en Córdoba. En esta ciudad, arrasada por las aguas, se produjeron numerosas víctimas fatales y pérdidas materiales. El pedido de auxilio a la CRA motivó un debate interno vinculado a estas nuevas funciones. La idea que primó fue que si había sido fundada para servir en las guerras internacionales o civiles, también lo era para actuar en las grandes epidemias y catástrofes. Se resolvió, por lo tanto, en el caso de Córdoba, el auxilio con provisiones así como la coordinación de su entrega.

Al mismo tiempo, el Comité porteño estimuló la emergencia de un subcomité de la $\mathrm{CR}$ en esa localidad para evitar desplegar desde Buenos Aires toda la atención y el auxilio solicitado. En Córdoba, los delegados aunaron las dispersas colaboraciones ofrecidas tanto por las organizaciones benéficas como por la población en general, formando un solo centro común para repartir con equidad los recursos recolectados en dinero y en especie. La CRA se puso a la cabeza en la articulación del accionar entre las entidades de beneficencia (ya fuesen éstas de orden católico o étnicas) y la población afectada, sin superponerse a las otras, pero con evidente interés de liderazgo. ${ }^{34}$

A partir de la participación en las inundaciones de Córdoba, la CRA colaboró en otras catástrofes ambientales, como el terremoto de la Rioja de 1894, a través de

32 Extraído de http://www.lagaceta.com.ar/nota/560830/sociedad/epidemia-1887.html

33 Boletín de la CRA, 1891, núm. 4: 6-8.

34 Boletín de la CRA, 1891, núm. 5. 
la comisión médica enviada por el Departamento Nacional de Higiene y con la remisión de carpas, ropas y víveres. También colaboró en 1899 con las víctimas de las inundaciones en General Roca (localizada en el entonces Territorio Nacional de Río Negro), a través de ropas y víveres distribuidos en Comités de Bahía Blanca, Patagones y Viedma. Igual situación sucedió un año después en Buenos Aires, cuando las aguas subieron y se debió socorrer a "centenares de familias", tanto habilitando un local como a través de ayuda monetaria para las víctimas (\$ 5000 m/n; Lozano 1932: 34-35).

La intervención de la CRA en la esfera civil se destacó por la aplicación de una serie de principios rectores, producto de su experiencia en el campo militar. Uno de sus roles fundamentales fue el de organizar las distintas logísticas de ayuda humanitaria para las víctimas de pestes, terremotos e inundaciones, ocupándose de centralizar y articular el auxilio brindado por otras organizaciones benéficas e incluso religiosas, mediante un conjunto de medidas destinadas a hacer efectiva especialmente la distribución de enseres, ropas y alimentos.

Nos parece significativo que en 1892 la entidad tomara contacto más directo con el Servicio de Sanidad del Ejército, e incluso participara de acciones en conjunto, con intercambio información y prácticas médicas que incluían movilización del personal. La CR debía ser un auxilio del Ejército en el caso de conflictos -no se indica si internos o externos-, cumpliendo una decidida acción patriótica, "proveyendo" (...) "al cuidado de nuestros bravos militares" (Lozano, 1932).

De acuerdo con Soprano (2018), el servicio se fundó en 1888 a través de la Ley Orgánica № 2.377 del Cuerpo de Sanidad del Ejército y la Armada, que otorgó estado militar a todo el personal de sanidad, y creó esa entidad en el Ejército y en la Armada, conformado por médicos, farmacéuticos y veterinarios. Esta ley se reglamentó en 1892 y 1896. En 1903, unas 258 personas (médicos y otro personal) formaban parte del servicio en las Fuerzas Armadas argentinas. Por entonces, además de la tarea en los hospitales militares y en los cuarteles con la atención de la tropa y oficiales, debían realizar inspecciones a un rango muy amplio de la ciudadanía, a raíz de la implementación de la ley de conscripción obligatoria en 1901, que obligaba a los jóvenes argentinos a instruirse en las armas para "servir a la Patria”. Los directores del servicio estaban al tanto de las modificaciones técnicas en la atención de heridos en caso de guerra y compartían espacios de formación con los miembros de la CR. Más adelante, como indicamos, formaron parte de sus autoridades de manera formal.

Otro aspecto tomado de la sanidad militar fue estimular la formación de las distintas sociedades en los estratos provinciales compuestas básicamente por voluntarios, lo que había sido una de las recomendaciones surgidas de la reunión preparatoria de la Convención de Ginebra, realizada en octubre de 1863. Se trató de un voluntariado que, en principio, convocaba a personas de diferente condición social, género, destinadas a colaborar bajo la supervisión de médicos. En el caso de la CRA, estuvo compuesta predominante por un sector socioeconómico alto. 
La intervención de la CRA en la esfera civil amerita contemplar también el impacto en las comunidades receptoras de su ayuda, pues engendró diversas reacciones: no siempre fue bienvenida su presencia y, en otras oportunidades, las acciones se interpretaron en clave política y no necesariamente humanitaria. El caso tucumano es un ejemplo de esa diversidad, ya que algunas memorias destacan que con la epidemia de cólera de 1886-87, la CRA fundó lazaretos, transportó enfermos abandonados y, con los carros recolectores de basura, pedían casa por casa camas para hospitalizar a los enfermos, todo lo cual fue apoyado por parte de la sociedad tucumana (Ponssa, 1940: 114). Sin embargo, hubo otras manifestaciones contrarias a su presencia, como la reacción social de 1887 en el suroeste tucumano, cuando un levantamiento de "montoneras" con gritos en contra de "gringos y masones" ocasionó la muerte de dos extranjeros y de un argentino de ascendencia francesa. Las tres personas asesinadas eran miembros de las Comisiones de Sanidad organizadas por la CRA (Goldman, 1990: 47).

La animosidad hacia los médicos se había desarrollado entre las capas más pobres de la campaña debido a las medidas implementadas para detener la epidemia. Blanqueos de casas, quema de ranchos y ropas, así como destrucción de sembradíos frutales, generaron la reacción contraria de una población que, además de la peste, veía destruidas sus pertenencias. El Cantar de las Montoneras recrea esos sentimientos: "Allá dentró la Cruz Roja / Contando que ella curaba / A los enfermos aún vivos / Así vivos los quemaba” (Goldman, 1990: 63).

Tal reacción social y enfrentamientos políticos pusieron en tensión las acciones de la CRA en Tucumán. El periódico El Orden identificaba a los voluntarios de esta entidad como aliados al gobierno nacional Juarista y, a la vez, opositores al gobierno provincial, culpabilizándolos de fomentar el malestar social (Gargiulo, 2011: 119; Goldman, 1990). En parte se explica esta acusación de parcialidad en que los tres miembros de la Comisión de Higiene eran masones, y en ese momento existía una imagen simbiótica entre Cruz Roja y masonería. Además había una propaganda conjunta de la iglesia y el grupo gobernante provincial contra los "gringos masones", que alimentó una inquina en ascenso y concluyó con el asesinato (Goldman, 1990: 69). Para conocer cuánto de esa impronta masónica era cierta, volveremos la mirada al interior de la CRA.

\section{Quiénes y cómo: los actores}

Una cuestión interesante de esta asociación fue la composición profesional y de género, y por otro lado su vinculación ideológica, al ser declaradamente una institución no religiosa. Veamos tal situación en 1891 y 1907, años sobre los cuales disponemos de información completa sobre la conformación de autoridades y socios, para observar las diferencias.

Inicialmente, la relación entre los aspectos bélicos de la $\mathrm{CR}$ y su vinculación con el honor y la batalla fortalecieron su masculinización; tal cuestión pudo observarse 
en otras entidades a nivel internacional y se replicó en la filial argentina. Del examen de los distintos comités en 1891 , se desprende que las mujeres eran una minoría: sobre 671 miembros de número, 593 (88\%) eran varones, y sólo 78 eran mujeres (12\%). ${ }^{35}$ Dentro de este conjunto sobresalen miembros de las familias más destacadas, sobre todo en la primera filial, que fue en la ciudad de Buenos Aires. Entre las mujeres se encontraban Cecilia Grierson, la primera médica argentina (Binda, Silveira y Kramer (2010), y Emma de la Barra, escritora que colaboró en numerosas revistas y autora de novelas. ${ }^{36}$ Además de estas dos integrantes, la composición femenina incluía apellidos de mujeres casadas y ocho "señoritas", cuyo común denominador parece ser la pertenencia a los núcleos de la élite, al menos al borde del cambio de siglo.

Igual situación se registra en el interior del país: en la composición del primer Consejo de Córdoba, en 1891, aparecen los médicos Garro, Olmedo, Vidal, Peña, Molina, Machado, Escalera, Del Campillo, Castellanos, García Montaña, Allende, Garzón Lezana, Moyano, Ponce, Del Viso, Deheza, Doering, Roca, Achaval y Funes, entre otros. ${ }^{37}$ Además del Consejo de Caballeros, se designó una Comisión de Damas, inicio de la participación de las mujeres en el gobierno de la Sociedad. La conformación de los distintos puestos de la CRA a lo largo de estas primeras décadas obedeció a la influencia central de facultativos y de otros integrantes, todos varones. Existiendo en esto a una lógica de subordinación de género propia de la composición de asociaciones de la época, las mujeres se integraron en comités aparte, situación que posteriormente se fue transformando, para "feminizar" casi por completo la asociación. En el caso de Córdoba, la Junta Directiva de la Sección Femenina estaba integrada por las matronas, familiares de los médicos. ${ }^{38}$

En 1907, se menciona para todo el país a 182 socios nuevos en la CRA -todos varones-, de los cuales 61 pertenecían a profesiones liberales (sobre todo médicos, pero también ingenieros, escribanos y militares), 17 hacendados y 64 comerciantes. ${ }^{39}$

Como Guillermo Rawson, reconocido masón, fue su primer presidente honorario, se alimentó la idea de que los destinos de la CRA estaban marcados por vínculos con esta organización, que ya gozaba de una larga trayectoria (Lazcano, 1927). De acuerdo a la bibliografía tradicional sobre la masonería, existen relaciones concretas en tal sentido, ya que Henri Dunant era masón y solicitó ayuda a todos los adeptos para difundir los principios y organizar esta asociación en diferentes países. Rawson, iniciado en la Logia Unión del Plata № 1 en Julio de 1856, respondió a esta convocatoria, al igual que otros miembros de la masonería local (Lappas, 1966: 328).

35 Boletín de la CRA, 1891, núm. 4: 37-46).

36 https://www.geni.com/people/Emma-de-la-Barra-Gonz\%C3\%A1lez/6000000026784074183

37 Boletín de la CRA, 1891, núm. 4: 6-8).

38 Se repiten los apellidos de Peñaloza, Soaje, Molina, Escalera, Deheza, Garzón y Castellanos, Allende, Caciro, Soria, Maceda, Argüello, Vélez, Roca y Urioste (Lozano, 1932: 29).

39 Boletín de la CRA, 1907, núm. 3: s/p; y Boletín de la CRA, 1907, núm. 4: 7. 
Si bien dentro de la CRA hubo masones, de los 671 miembros con que contaba en 1891, sólo 33 (5\%) eran adeptos a esta organización. A nivel cualitativo la situación puede haber sido diferente, dado el peso cultural y social de quienes la integraron. Por ejemplo, uno de ellos fue Samuel Gache, doctor en medicina, iniciado en 1872 en la Logia Consuelo del Infortunio. Distinguido higienista y especialista en tuberculosis, presidió el Círculo Médico Argentino, publicó varias obras científicas y colaboró en diversos diarios y revistas (Lappas, 1966: 200). A esa misma Logia pertenecieron Ignacio Pirovano, médico que se había perfeccionado en Francia y reconocido catedrático de la Facultad de Medicina de la Universidad de Buenos Aires (Lappas, 1966: 316), y Juan Argerich, catedrático y director de la Casa de Niños Expósitos. A ellos se sumaban Manuel Augusto Montes de Oca, médico, director de hospitales, afiliado a la Logia Confraternidad Argentina № 2 de Buenos Aires; José María Ramos Mejía, reconocido médico y funcionario, Director de la Asistencia Pública, presidente del Departamento Nacional de Higiene, Diputado Nacional en varios períodos y prolífero escritor, iniciado en la Logia Docente (Lappas, 1966: 327); Eliseo Cantón, decano de la Facultad de Medicina, miembro de la Academia de Medicina, Diputado Nacional y miembro de la Logia Bernardino Rivadavia, entre otros (Lappas,1966: 144). Es decir, se trataba de figuras públicas reconocidas por su labor médica y con destacada presencia política en diferentes áreas gubernamentales, lo que provocó que desde la literatura masónica se alimentara el vínculo entre ambas instituciones.

Pero también la CRA contó con personajes cuyos destinos no estaban vinculados a los masones, como Lucio Meléndez, catedrático Director del Hospicio de las Mercedes, precursor de la medicina forense (Di Liscia, 2002: 197); Guillermo Udaondo, quien fuera médico y gobernador de Buenos Aires entre 1894-1898 (Cutolo, 1968); y Tomás de Anchorena, abogado, Ministro de Relaciones Exteriores y del Interior durante la presidencia de Luis Sáenz Peña (1892-1895). Por lo tanto, durante sus primeros años, en la CRA convivieron diversas tendencias políticas e ideológicas, cuyo denominador común era la pertenencia a los sectores más encumbrados de la sociedad porteña y con influencia política determinante en los destinos nacionales.

\section{A modo de cierre}

En las páginas precedentes se analizan el origen y las primeras décadas de vida de la CRA, mostrando los diferentes intereses por los que estuvo cruzada esta organización internacional de tipo humanitario en la Argentina. Fue precisamente su carácter internacional lo que la distinguió del resto de las organizaciones de beneficencia y/o filantrópicas existentes en el país.

Sus principios se remontaban al Convenio de Ginebra, que sentó las bases del desarrollo del derecho humanitario contemporáneo. Sus características principales eran establecer normas permanentes, escritas, de alcance universal, destinadas a proteger a las víctimas de los conflictos mediante la neutralidad de la entidad. Luego, para el periodo bajo estudio, se firmaron otros tratados en 1868, 1899, 1906 y 1907, fruto de diversos encuentros y congresos internacionales. 
En este sentido, podríamos decir que el trabajo ilumina sobre los sucesos, conflictos ideológicos y tensiones sociales por los que estuvo atravesada la aplicación de esa primera norma vinculada con el origen del derecho humanitario en nuestro país. No se trató de un proceso lineal y progresivo, ni tuvo el acuerdo de todos los sectores sociales (en especial de las élites), a pesar de sus objetivos altruistas. En distintas oportunidades se puso en tela de juicio la neutralidad de la entidad, caja de resonancia de los conflictos políticos internos. La intervención de la CRA en diferentes movimientos revolucionarios provinciales, entre finales del siglo XIX y primera década del XX, podría haber además incluido un apoyo encubierto a una de las facciones en pugna, lo que también ocurrió cuando se trató de ayuda internacional. Tal fue el caso del intento de auxilio brindado a Uruguay en relación a la Revolución de 1904, el envío de un buque con elementos sanitarios "motivó un enojoso entredicho, que sin tener ulteriores consecuencias, permitió apreciar que era indispensable establecer claramente (ese auxilio) por un convenio internacional" (Lozano, 1932: 35). La situación, observada diplomáticamente por Lozano, uno de los portavoces de la institución y por entonces ya reconocido sanitarista, implicaría que la "ayuda" se dirigió quizás a la facción opositora al gobierno triunfante en las elecciones de 1903. Dado que ese movimiento organizado por el Partido Blanco, iba en contra del gobierno José Batlle y Ordóñez, líder del Partido Colorado y gestor con posterioridad de importantes reformas sociales en Uruguay (Oddone, 1992: 127), es probable que la intervención de una entidad argentina haya sido interpretada como de apoyo a los anti-batllistas. Los uruguayos, además, ya contaban desde 1897 con una filial de la CR en su país, a la que según Lozano habrían contribuido a fundar desde Argentina (Lozano, 1932: 31).

La particularidad de la intervención en casos de epidemias y catástrofes naturales merece un análisis diferente, ya que se intentó asumir un rol preponderante a nivel de estrategia organizacional (con el suministro de víveres o ropas), así como estructurar la ayuda médica y la provisión de recursos sanitarios. Justamente, muchas de las áreas devastadas por desastres o donde la población había sido diezmada por epidemias, carecían de un sistema médico-sanitario organizado, o bien estaba muy por debajo de las necesidades requeridas por una demografía en expansión (por ejemplo, en los barrios de la Capital Federal). En tal caso, esas emergencias proporcionaron el puntapié inicial para la programación de filiales de la CRA o, si existían previamente, las fortalecieron con nuevos socios y un ímpetu transformador útil como base para acciones sanitarias posteriores. A partir de 1890, el Consejo Supremo de la CRA se esforzó por obtener un reconocimiento nivel nacional para luego poder conformar bajo su control la federación de comités y sub-comités en localidades de diversas provincias argentinas.

Dado el papel y el status de los médicos en el país, estos profesionales se preocuparon por su creación, paralelamente a la influencia de asociaciones formadas por notables que, a la vez, eran inmigrantes recientes. La CRA fue una instancia más de un sector de médicos jóvenes para posicionarse dentro del campo médico local, y así pujar por hacerse lugar en las decisiones políticas y/o profesionales del escenario nacional. En relación a las ideologías y pertenencias partidarias de los miembros del Comité Central, se trataba de un grupo heterogéneo; muchos de 
ellos pertenecían a diferentes logias masónicas, lo que no fue condicionante de las decisiones de la CRA. El grupo de socios que conformaron los comités a lo largo de estas primeras décadas obedeció a la influencia central de facultativos y de otros integrantes, pertenecientes a las clases con mayores recursos, y en el caso de las comisiones directivas y del Comité Central, todos fueron varones. Obedeciendo en esto a una lógica de subordinación de género, propia de la composición de asociaciones de la época, las mujeres se integraron en comités aparte, situación que posteriormente se fue transformando, para "feminizar" la CRA casi por completo. En sus inicios, la sensibilidad, el cuidado y la abnegación, valores supuestamente femeninos, no estaban en sintonía con una asociación que proponía reglas de juego claras para moderar los efectos de la guerra, tarea que en ese imaginario era masculina por excelencia.

La participación activa de la CRA en casos de calamidades públicas también fortalecía a los médicos, sector profesional que intentó concentrar la tarea de regular las acciones estatales en relación a la salud de la población, avanzando sobre el Estado (Di Liscia, 2017). En esta institución, fue sin embargo relevante la tarea de otros profesionales, como los ingenieros, y la participación de empresarios y comerciantes, que durante este período permitieron mantener cierta solvencia económica.

En 1914 se inició para la CRA una etapa diferente a los cincuenta años anteriores, donde se había logrado legitimidad y anudado lazos entre sectores diversos para su organización, además de cierta autonomía económica. Cuando se produjo una conflagración de características mundiales, la misma entidad tuvo que reorganizarse, dado que volvieron a florecer las diferencias nacionales. El caso argentino resulta de gran interés, dentro del conjunto de las $\mathrm{CR}$ americanas, por la cantidad de población extranjera existente por entonces, y porque se hallaban en su territorio diferentes asociaciones de los países en guerra, que le imprimieron una lógica nacionalista, ajena a los principios de imparcialidad y humanitarismo. Tal situación permite comprender la enorme riqueza del estudio de una asociación de estas características para nutrir la historiografía argentina y la historia misma de las filiales de la CR. 


\section{Bibliografía}

" Aráoz Alfaro, G. (1938). Crónicas y estampas del pasado. Buenos Aires: El Ateneo.

" Alvarez A. (2007). De la Higiene Pública a la Higiene Social en Buenos Aires, una mirada a través de sus protagonistas, 1880-1914. Boletín Mexicano de Historia y Filosofía Médica 10 (1): 4-11.

" Biernat, C., Ramacciotti, K. et al, (Dirs.) (2015). La salud pública y la enfermería en la Argentina, Bernal: Universidad Nacional de Quilmes.

" Binda, M. del C., Silveira, C. y Kramer, C. (2010). Cecilia Grierson, la primera médica argentina". Revista Argentina de Radiología. 74 (4): 361-364.

" Cantón, E. (1982). Informe del Inspector General, referente a un asunto de interés público". Boletín de la Cruz Roja Argentina, núm. 11: 7-9.

" Carbonell, M. (1953). La personalidad científica de Rawson. En Palcos, A. (Dir.) Guillermo Rawson. Escritos científicos. Colección: Grandes Escritores Argentinos. Buenos Aires: Jackson.

"Coni, E. (1918). Memorias de un médico higienista. Contribución a la historia de la Higiene Pública y Social Argentina (1867-1917). Buenos Aires: Ediciones Asociación Médica Argentina, Tomo 1.

" Cruz Roja Argentina (1891-1908). Boletín de la CRA, órgano oficial, Buenos Aires (serie incompleta: 1891-1908).

" Cruz Roja Argentina (1919). Cruz Roja Argentina, Su programa para épocas de paz. Buenos Aires: Imprenta Estrach.

"Cutolo, V. O. (1968). Nuevo diccionario biográfico argentino (1750-1930). Buenos Aires: Elche.

"Di Stefano, R., Sábato, H. Romero J. L. y Moreno, J.L (2002). De las cofradías a las organizaciones de la sociedad civil. Historia de la iniciativa asociativa en Argentina, 17761990. Buenos Aires: Gadis.

" Di Liscia, M. S. (2002). Cuerpos para experimentar. Objetivación médica, positivismo y eliminación étnica en Argentina (1860-1890). Revista Asclepio 54 (1): 183-200.

"Di Liscia, M.S. (2017). Del brazo civilizador a la defensa nacional: Políticas sanitarias, atención médica y población rural (Argentina, 1900-1930). Historia Caribe, Universidad del Atlántico, Vol. 12, núm. 31. DOI: http://dx.doi.org/10.15648/hc.31.2017.6, 2017.

" Dunant, H. (1982). Recuerdo de Solferino. Madrid: Comité Internacional de la Cruz Roja.

"Eleta, F. (2017). La medicina en el Ejército de los Andes. Revista de la Asociación Médica Argentina, Vol. 130, núm. 2: 32-40.

"Fernández, A. (2016). Persistencias y rupturas en la identidad española de los intelectuales catalanes de Buenos Aires (1850-1950). Anuario IEHS, 31 (2) 16: 85-102.

" Fernández, M. E. coord. (2017). La salud: instituciones, espacios y actores. Buenos Aires: Imago Mundi.

" Fleck, L. (1989). La génesis y el desarrollo de un hecho científico. Madrid: Alianza Universidad.

" Flores García F. (2017). Siluetas revolucionarias: Jose Paul y Angulo. Diario Blanco y Negro, 13 de febrero de 1916: 3. Consultado el 22/04/2017 en: http://hemeroteca.abc. 
es/nav/Navigate.exe/hemeroteca/madrid/blanco.y.negro/1916/02/13/030.html

" Fustioni O. (1971). El medio social finisecular y los escritores médicos argentinos. Boletín de la Academia Nacional de Medicina. Vol. 49, semestre 1: 130-143.

" Gallo, E. (1992). Política y Sociedad en Argentina, 1870-1916. Bethell L. (ed) Historia de América Latina. Vol. 10. Barcelona: Cambridge University Press-Editorial Crítica: 41-64.

" García Sebastiani, M. (2010). Patriotas entre Naciones. Elites emigrantes españolas en Argentina (1870-1940). Madrid: Editorial Complutense.

" Gargiulo, M. C. (2011). El cólera: oportunidades de control y resistencias populares. Tucumán 1886-1887. Estudios Sociales, Vol. 41, núm. 3: 97-125.

" González Leandri, R. (1999). Curar, persuadir, gobernar. La construcción histórica de la profesión médica en Buenos Aires. 1852-1886. Madrid: CSIC, Madrid.

" Goldman, N. (1990). El levantamiento de Montoneras contra gringos y masones en Tucumán, 1887. Tradición oral y cultura popular. Boletín del Instituto de Historia Argentina y Americana Dr. Emilio Ravignani, 2 (1), pp: 44-74.

" Hobsbawm, E. y T. Ranger (eds.). (2002) La invención de la tradición. Barcelona: Crítica.

" Hutchinson, J.F. (1996). Champions of Charity. War and the Rise of the Red Cross. Boulder: Westview Press.

" Manzella M. et al (1980). Historia de la CRA, 1ำ centenario 1980.Buenos Aires: Consejo Supremo de la Cruz Roja.

" Manzella M. (1982). La Cruz Roja. Pasado, presente y futuro. Su mística y su tónica. La guerra y la Paz, Buenos Aires: Consejo Supremo de la Cruz Roja.

" Meyer, M (2009). The importance of the International Conference of the Red Cross and Red Crescent to National Societies: fundamental in theory and in practice. International Review of the Red Cross. Vol. 91, núm. 876: 713-732.

" Martínez A. B. (1891). Guillermo Rawson. Buenos Aires: Compañía Sudamericana de Billetes de Banco.

" Muñoz Blanco, M. E. (1999). Cruz Roja Española. Un estatuto jurídico singular. Madrid: Tecnos.

" Landa, N. (2016). Muertos y heridos, y otros textos. Selección y estudio introductorio: Guillermo Sánchez y Jon Arrizabalaga, Navarra: Pamiela.

" Lappas, A. (1958). La masonería argentina a través de sus hombres, Buenos Aires: Establecimiento Gráfico de R. Riego.

" Larrain, J. (1895). Biografía del Dr. Guillermo Rawson. Buenos Aires: Imprenta de Solá y Hermanos.

" Lazcano, M. (1927). Las sociedades secretas, políticas y masónicas. Buenos Aires: El Ateneo.

" Lobato, M. Z. (2000). Estado, gobierno y política en el régimen conservador. Lobato, M. Z. dir. El progreso, la modernización y sus límites (1880-1916). Nueva Historia Argentina. Tomo V. Buenos Aires, Sudamericana: 179-208.

" Lozano, N. (1932). El Libro de la Cruz Roja Argentina. Publicación Oficial. Buenos Aires: Editorial Imprenta Talleres Gráficos Fabril Financiera.

" Oddone, J. A. (1992). La formación del Uruguay moderno, c. 1870-1930. Bethell, L. ed. Historia de América Latina, T. 10: América del Sur, 1870-1930. Barcelona: Cambridge University Press-Editorial Crítica: 135-156. 
"Pesqueira, J. (1919). Historia de la Asociación Española de Socorros Mutuos de Buenos Aires. Revista Mensual de la Asociación Española de Socorros Mutuos de Buenos Aires, Año VIII, núm. 82, Buenos Aires, 1919. Consultado el 23/04/2017 en: http://staging.ilo. org/public/libdoc/historical/1901-2000/73603.pdf.

" Piccinali, H. J. (1981). Vida del teniente general Nicolás Levalle. Buenos Aires: Círculo Militar.

" Ponssa, J. (1940). Una epidemia de cólera morbo azotó a Tucumán hace 50 años. Tribuna Farmacéutica, 2.

"Prieto, A. (1996). Rosario: epidemias, higiene e higienistas en la segunda mitad del siglo XIX. Lobato, M. Z. ed. Política, médicos y enfermedades. Lecturas de historia de la salud en la Argentina. Buenos Aires, Biblos: 57-73.

" Rawson, G. (1891). Escritos y Discursos. Buenos Aires: Compañía Sudamericana de Billetes de Banco, Tomos I y II.

" Roberts, P. (1880). Memoria presentada a los miembros de la Asociación Internacional de la Cruz Roja (Argentina). Buenos Aires: Imprenta Europea.

"Sábato, H. (2008). Buenos Aires en armas. La revolución de 1880. Buenos Aires: Siglo XXI Editores.

"Serrano, M. A. (1995). La capitalización de Buenos Aires. Buenos Aires: Editorial Círculo Militar Argentino.

"Soprano, G. (2018). El servicio de sanidad militar en el proceso de modernización, burocratización y profesionalización del ejército argentino (1888-1938). Presentado para evaluación en Salud Colectiva, UNL.

"Souza, P. (2004). El Círculo Médico Argentino y su papel como partido científico en la historia de la medicina argentina. Buenos Aires 1875-1883. Saber y Tiempo, Vol. 6, núm. 22: 107-140.

"Veronelli, J. C. y Veronelli Correch, M. (2004). Los orígenes de la salud pública en la Argentina. Buenos Aires: Organización Panamericana de la Salud, Tomo I.

"Visacovsky, S. (2005). El temor a escribir sobre historias sagradas. Memoria social, moralidad política y audiencias nativas en la Argentina. Frederic, S. y Soprano, G. (comp.) Cultura y política en etnografías sobre Argentina. Bernal, UNQUI: 271-315. 